\title{
TWO NOTES ON IMBEDDED PRIME DIVISORS
}

\author{
L. J. RATLIFF, JR.
}

(Communicated by William C. Waterhouse)

\begin{abstract}
The first note shows that if $R<T$ are any two Noetherian rings, then there exists a Noetherian ring $B$ between $R$ and $T$ which has a maximal ideal $N$ such that $\operatorname{grade}(N) \leq 1$ and $N \cap R$ is a maximal ideal. The second note shows that if $R$ is a Noetherian ring, then there exists a free quadratic integral extension ring $B$ of $R$ such that $\operatorname{Spec}(B) \cong \operatorname{Spec}(R)$ and such that if $I$ is any regular ideal in $R$ and $P_{1} \cap \cdots \cap P_{g}$ are prime ideals in $R$ containing $I$, then there exists an ideal $J$ in $B$ integrally dependent on $I B$ such that the prime ideals corresponding to the $P_{i}$ are prime divisors of $J^{n}$ for all $n \geq 1$.
\end{abstract}

1. Introduction. The two results mentioned in the abstract were motivated by the following two problems:

(a) If $R<A \subseteq R^{\prime}$, the integral closure of $R$, are local domains such that $\operatorname{Spec}(R) \cong \operatorname{Spec}\left(R^{\prime}\right)$, if $Q$ is a prime ideal in $A$ such that $\operatorname{grade}\left(Q A_{Q}\right)=1$, and if $P=Q \cap R$, then must it be true that grade $\left(P R_{P}\right)=1$ ?

(b) If $P_{1}, \ldots, P_{g}$ are regular prime ideals in a Noetherian ring $R$, then does there exist an ideal $I$ in $R$ such that the $P_{i}$ are the prime divisors of $I^{k}$ for all large $k$ ?

The first problem was mentioned in $[\mathbf{1 3},(6.7 .1)]$ (and it is shown [10, (33.11)], even without assuming that the spectra are isomorphic, that the answer is yes when $A=R^{\prime}$ ), and (b) was suggested by Theorem 21 on p. 230 in [14], where it is shown that there exists an ideal $I$ such that $\operatorname{Ass}(R / I)=\left\{P_{1}, \ldots, P_{g}\right\}$.

In $\S 2$ the first result mentioned in the abstract is proved, and it immediately follows from this that the answer to (a) is no. Then $\S 2$ is closed by using this result to show that there exists an infinite chain of local domains $L_{0}<L_{1}<\cdots$ such that there exists a height one principal prime ideal $p$ in $L_{0}$ such that $p L_{2 n}$ is a principal prime ideal and $p L_{2 n+1}$ has an imbedded prime divisor for $n=0,1, \ldots$

Problem (b) is briefly considered in $\S 3$, and it is shown that the answer to this problem is also no, but there always exist ideals $I$ such that the $P_{i}$ are among the prime divisors of $I^{k}$ for all $k \geq 1$. Then the following more difficult question is considered: If $I$ is a regular ideal contained in $\bigcap\left\{P_{i} ; i=1, \ldots, g\right\}$, then does there exist an ideal $J$ integrally dependent on $I$ such that $\left\{P_{i} ; i=1, \ldots, g\right\} \subset \operatorname{Ass}\left(R / J^{k}\right)$ for all $k \geq 1$. It is shown that the answer is no, but the second result mentioned in the abstract shows that this does hold in some free quadratic integral extension ring $B$ of $R$ such that $\operatorname{Spec}(B) \cong \operatorname{Spec}(R)$.

Received by the editors August 4, 1986.

1980 Mathematics Subject Classification (1985 Revision). Primary 13A15; Secondary 13B20.

Key words and phrases. Cohen-Macaulay ring, flat extension ring, grade of an ideal, integral closure of an ideal, integral extension ring, Notherian ring, prime divisor semilocal ring.

Research on this paper was supported in part by National Science Foundation Grant DMS8521058 . 
For the most part our methods are elementary. The main results are not profound, but they were somewhat of a surprise to me; for some reason I thought prime divisors would behave better under integral extensions.

2. On the ubiquity of grade one maximal ideals. If $R<T$ are Noetherian domains, then it follows from (2.1) that there always exists an intermediate Noetherian domain with a maximal ideal of grade one which lies over a maximal ideal in $R$. This result explains the title of this section.

The proof of (2.1) shows somewhat more than is stated in the theorem, as is explained in (2.2). It is for this reason that the $\operatorname{ring} A$ is used in the second (resp., third) paragraph of the proof of (2.1) instead of the ring $C+Q T$ (resp., $R+M T)$.

(2.1) THEOREM. Between any two distinct Noetherian rings $R<T$ there exists an intermediate ring $B$ which is finitely generated over $R$ and which has a maximal ideal $N$ such that $\operatorname{grade}(N)=\operatorname{grade}\left(N B_{N}\right) \leq 1$ and $N \cap R$ is a maximal ideal.

PROOF. It clearly suffices to prove the theorem under the assumption that $T=R[t]$ is a simple extension ring of $R$, and since $N$ is maximal it is clear that $\operatorname{grade}(N) \leq 1$ if and only if $\operatorname{grade}\left(N B_{N}\right) \leq 1$.

If $t$ is transcendental over $R$, then $T=R[t]$ is integral over $C=R\left[t^{2}\right]$. Let $Q=\left(M, t^{2}\right) C$, where $M$ is a maximal ideal in $R$, so $Q$ is a maximal ideal in $C$ that lies over $M$. Also, $T_{C-Q}$ is a finite $C_{Q}$-algebra that properly contains $C_{Q}$, so by the Lemma of Krull-Azumaya $[\mathbf{1 0},(4.1)]$ it follows that $C_{Q} \subseteq(C+Q T)_{C-Q} \subseteq$ $C_{Q}+Q C_{Q} T_{C-Q}<T_{C-Q}$, so $C+Q T<T$. Therefore, since $C$ is Noetherian and $T=R[t]$ is a finite $C$-module, there exists a (necessarily finite) $C$-algebra $A$ which is a maximal proper subring of $T$ containing $C+Q T$. Then $A$ is finitely generated over $R$, and it is clear that $Q T$ is contained in the conductor $Y=A: T$ of $A$ in $T$ and that if $P \in \operatorname{Spec}(A)$ is a minimal prime divisor of $Q T$, then $P$ is a maximal ideal in $A$ that lies over $Q$. Also, $t^{2} \in Q \subseteq Y \subseteq P$, so grade $(P) \geq 1$, since $t^{2}$ is regular in $T$ and $P<A<T$, and $Y t \subseteq Y$, so $t^{3} \in Y \subseteq A$. Therefore $Y\left(t^{3} / t^{2}\right)=Y t \subseteq A$, so $Y \subseteq t^{2} A: t^{3} A<A$; it follows that grade $(P)=1$. Also, $P \cap R=M$, so the conclusion follows with $B=A$ and $N=P$. Therefore it may be assumed that $t$ is algebraic over $R$.

If $t$ is integral over $R$, then let $M$ be a maximal ideal in $R$ such that $R_{M}<T_{R-M}$. (Note that $R: T \neq R$ since $R<T$, so there exists a maximal ideal $M$ in $R$ such that $R: T \subseteq M$. Then, since $T$ is a finite $R$-module, it follows from [10, (18.1)(2)] that $R_{M}: T_{M}=(R: T)_{M} \subseteq M R_{M}$, so $R_{M}<T_{R-M}$.) Then it follows as in the preceding paragraph that there exists a finite $R$-algebra $A$ which is a maximal proper subring of $T=R[t]$ containing $R+M T$ and that every minimal prime divisor $P$ of $Y=A: T$ is a maximal ideal in $A$ that lies over $M$. Therefore for each $b \in Y$ there exists $c \in A$ such that $b t=c$, since $Y t \subseteq A$. If $Y T \subseteq \bigcup\{z ; z \in \operatorname{Ass}(T)\}$, then it follows that $P T$ is contained in some prime divisor $z$ of zero in $T$. But then by integral dependence $z$ must be a maximal ideal in $T$, grade $(z)=0$, and $z \cap R$ is maximal, so the conclusion follows with $B=T$ and $N=z$. Therefore it may be assumed that there exists $b \in Y$ such that $b$ is regular in $T$, so $t=c / b$, where $b t=c$. Then $Y(c / b) \subseteq A$, so it follows as in the preceding paragraph that $Y \subseteq b A: c A<A$, so grade $(P)=1$; the conclusion follows with $B=A$ and $N=P$. Therefore it may be assumed that $t$ is algebraic and not integral over $R$. 
Let $C$ be the integral closure of $R$ in $T$. If there exists $c \in(C-R)$, then $R[c]$ is integral over $R$ and $R[c] \subseteq T$, so the conclusion follows as in the preceding paragraph with $R[c]$ in place of $T=R[t]$. Therefore it may be assumed that $R$ is integrally closed in $T$.

Let $p^{\prime}$ be a prime ideal in $T$ and assume that the $p^{\prime}$-residue class of $t$ is transcendental over $R / p$, where $p=p^{\prime} \cap R$. Then $\operatorname{Ker}(R[X] \rightarrow T) \subseteq p R[X] \subseteq M R[X]$, where $M$ is a maximal ideal in $R$ containing $p$, so $M T$ is a depth one prime ideal and the $M T$-residue class of $t$ is trancendental over $R / M$. Also, $T$ is integral over $R\left[t^{2}\right]$, so it follows that $M R\left[t^{2}\right]$ is a depth one prime ideal and the $M R\left[t^{2}\right]$-residue class of $t^{2}$ is transcendental over $R / M$. Therefore $R \subseteq R\left[t^{2}\right]<R[t]=T$, since $R\left[t^{2}\right] / M R\left[t^{2}\right]<R[t] / M R[t]$, and $Q=\left(M, t^{2}\right) R\left[t^{2}\right]$ is a maximal ideal in $R\left[t^{2}\right]$ that lies over $M$ and $R\left[t^{2}\right]_{Q}<R[t]_{R\left[t^{2}\right]-Q}$. Therefore the conclusion follows as in the third paragraph of this proof with $R\left[t^{2}\right]$ and $Q$ in place of $R$ and $M$, respectively.

Therefore it may be assumed that, for each $p^{\prime} \in \operatorname{Spec}(T)$, the $p^{\prime}$-residue class of $t$ is algebraic over $R /\left(p^{\prime} \cap R\right)$. But this implies that each $p^{\prime} \in \operatorname{Spec}(T)$ is both maximal and minimal in the set of prime ideals in $T$ that lie over $p^{\prime} \cap R$. Therefore by the Peskine-Evans version of Zariski's Main Theorem [2] it follows that

$$
\text { for each } p^{\prime} \in \operatorname{Spec}(T), T_{p^{\prime}}=R_{p^{\prime} \cap R} \text {. }
$$

Let $Z=\operatorname{Rad}(T)$ and let an overbar denote residue class modulo $Z$. Then $Z=$ $\operatorname{Rad}(R)$, since $R$ is integrally closed in $T$, so it readily follows that $\bar{R}<\bar{T}$, that $\bar{R}$ is integrally closed in $\bar{T}$, and that $(*)$ holds for $\bar{R}$ and $\bar{T}$ in place of $R$ and $T$, respectively. It then follows that $\bar{R}$ and $\bar{T}$ have the same total quotient ring, namely $\bigoplus\left\{R_{z} / z R_{z} ; z\right.$ is a minimal prime ideal in $\left.R\right\}$, so there exist $\bar{b}, \bar{c} \in \bar{R}$ such that $\bar{b}$ is regular and $\bar{t}=\bar{c} / \bar{b}$. To complete the proof it will first be shown that there exists a maximal ideal $\bar{M}$ and $\bar{R}$ such that $\bar{R}_{\bar{M}}<\bar{T}_{\bar{R}-\bar{M}}, \bar{b}^{*}, \bar{c}^{*}$ are regular in $\bar{R} \bar{M}$, and $\bar{t}^{*}=\bar{c}^{*} / \bar{b}^{*}$, where the $*$ denotes image in $\bar{T} \bar{T}-\bar{M}$.

For this, note that $\bar{T}=\bigcap\left\{\bar{T}_{\left[\bar{M}^{\prime}\right]} ; \bar{M}^{\prime}\right.$ is a maximal ideal in $\left.\bar{T}\right\}$, where $\left[\bar{M}^{\prime}\right]$ denotes the set of regular elements in $\bar{T}-\bar{M}^{\prime}$, and a similar statement holds concerning $\bar{R}$, so since $\bar{R}<\bar{T}$ it follows from (*), applied to $\bar{R}$ and $\bar{T}$ in place of $R$ and $T$, that there exists a maximal ideal $\bar{M}$ in $\bar{R}$ that is lost in $\bar{T}$; that is, there exists no prime ideal in $\bar{T}$ that lies over $\bar{M}$. Fix such an ideal $\bar{M}$ and let $S=\bar{R}-\bar{M}$. Then $\bar{R}_{\bar{M}}<\bar{T}_{S} \subseteq\left(\bar{R}_{\bar{b}}\right)_{S}$ (where $\bar{t}=\bar{c} / \bar{b}$ ), and the image of $\bar{b}$ in $\bar{R}_{\bar{M}}$ is regular, so $\bar{R}_{\bar{M}}$ and $\bar{T}_{S}$ have the same total quotient ring and $\bar{T}_{S}=\bar{R}_{\bar{M}}\left[\bar{c}^{*} / \bar{b}^{*}\right]$. Therefore, for each $\bar{z} \in \operatorname{Ass}\left(\bar{R}_{\bar{M}}\right)$ it holds that $\bar{z}^{\#}=\bar{z}\left(\bar{R}_{\bar{b}}\right)_{S} \cap \bar{T}_{S} \in \operatorname{Ass}\left(\bar{T}_{S}\right)$ and $\bar{R} \bar{M} / \bar{z}<\bar{T}_{S} / \bar{z}^{\#}$, since $\bar{M} \bar{R}_{\bar{M}}$ is lost in $\bar{T}_{S}$, so $\bar{c}^{*} \notin \bar{z}^{\#}$, and so $\bar{c}^{*}$ is regular in $\bar{R} \bar{M}$.

Also, $\bar{b}^{*}$ is regular in $\bar{R}_{\bar{M}}$, so $\bar{b}^{*} / \bar{c}^{*}$ and $\bar{c}^{*} / \bar{b}^{*}$ are in the total quotient ring of $\bar{R}_{\bar{M}}$. Let $D$ be the integral closure of $\bar{R}_{\bar{M}}$, so $D$ is the direct sum of the integral closures of the rings $\bar{R}_{\bar{M}} / \bar{z} \bar{R}_{\bar{M}}$, where $z$ is a minimal prime ideal in $R$ that is contained in $M$. Since $\bar{M} \bar{R} \bar{M}$ is lost in $\bar{T}_{S}$ it is clear that $\bar{t}^{*}=\bar{c}^{*} / \bar{b}^{*} \notin D_{\left[N^{\prime}\right]}$ for all maximal ideals $N^{\prime}$ in $D$. But if neither $\bar{c}^{*} / \bar{b}^{*}$ nor $\bar{b}^{*} / \bar{c}^{*}$ are in $D_{\left[N^{\prime}\right]}$, then it follows from [15, Corollary, p. 20] that $N^{\prime} D_{\left[N^{\prime}\right]}\left[\bar{c}^{*} / \bar{b}^{*}\right]$ is a depth one prime ideal, and then by integral dependence it follows that $\bar{M} \bar{T}_{S}$ is a depth one prime ideal. Therefore, necessarily $\bar{b}^{*} / \bar{c}^{*} \in D_{\left[N^{\prime}\right]}$ for all maximal ideals $N^{\prime}$ in $D$, so $\bar{b}^{*} / \bar{c}^{*} \in D$. It then follows that $\bar{b}^{*} / \bar{c}^{*}$ is integral over $\bar{T}_{S}$ and $\bar{c}^{*} / \bar{b}^{*} \in \bar{T}_{S}$, so $\bar{b}^{*} / \bar{c}^{*} \in \bar{T}_{S}$. But $\bar{R}_{\bar{M}}$ is 
integrally closed in $\bar{T}_{S}$, since $\bar{R}$ is integrally closed in $\bar{T}$, so $\bar{b}^{*} / \bar{c}^{*} \in \bar{R} \bar{M}$, and so $\bar{T}_{S}=\left(\bar{R}_{\bar{M}}\right)_{\bar{b}^{*} / \bar{c}^{*}}$ is a localization of $\bar{R}_{\bar{M}}$. If height $(\bar{M})>1$, then let $\bar{d} \in \bar{M}$ such that height $\left(\left(\bar{b}^{*} / \bar{c}^{*}, \bar{d}^{*}\right) \bar{R}_{\bar{M}}\right)=2$ and let $y=\bar{d}^{*} /\left(\bar{b}^{*} / \bar{c}^{*}\right)$. Then $\bar{R}_{\bar{M}}[y] \subseteq \bar{T}_{S}, \bar{M} \bar{R}_{\bar{M}}[y]$ is a depth one prime ideal, and the $\bar{M} \bar{R} \bar{M}[y]$-residue class of $y$ is transcendental over $\bar{R}_{\bar{M}} / \bar{M} \bar{R}_{\bar{M}}$. Let $x \in T$ such that $\bar{R}[\bar{x}]_{S}=\bar{R}_{\bar{M}}[y]$. Then it readily follows that $Z=Z R[x] \subseteq M R[x]$, that $M R[x]$ is a depth one prime ideal, and that the $M R[x]$-residue class of $x$ is transcendental over $R / M$, so the conclusion follows as in the fifth paragraph of this proof with $R[x]$ in place of $T=R[t]$.

Therefore it may be assumed that height $(\bar{M})=1$. (Note that $\bar{b}$ is regular in $\bar{R}$ and necessarily $\bar{b} \in \bar{M}$, since $\bar{T}=\bar{R}[\bar{c} / \bar{b}]$ and $\bar{M}$ is lost in $\bar{T}$, so height $(\bar{M}) \geq 1$.) Then there exists a minimal prime ideal $z$ in $R$ such that $z<M$ and height $(M / z)=$ 1. Therefore either $M \in \operatorname{Ass}(R)$, so $\operatorname{depth}(M)=0$, or $M$ is a minimal prime divisor of $(z, e) R$ for some regular element $e \in M$, so $\operatorname{depth}(M)=1$, by [15, Lemma 1 , p. 394]. Thus, in either case, the conclusion holds with $B=R$ and $N=M$. Q.E.D.

In [1], rings $A<B$ such that there exist no rings properly between them are called adjacent, and therein and in [3] many properties of such rings are proved. Also, the results in [8] are related to adjacent rings, and in particular it is shown in [8, Theorem 1] that $A: B$ is a maximal ideal in $A$ when $A<B$ are adjacent and $B$ is integral over $A$. (2.2) shows that if $R<T$ are Noetherian rings, then there usually are integrally dependent adjacent Noetherian rings between $R$ and $T$.

(2.2) REMARK. If $R<T$ are Noetherian rings, then either: (a) There exist adjacent Noetherian rings $A<B$ between $R$ and $T$ such that: $A$ is finitely generated over $R ; B$ is integral over $A$; and $A: B$ is a maximal ideal in $A$ that lies over a maximal ideal in $R$ and is such that either grade $\left(M^{\prime}\right)=0$ for some maximal ideal $M^{\prime}$ in $B$ lying over $A: B$ or grade $(A: B) \leq 1$; or, (b) $R$ is integrally closed in $T$ and $R / Z$ is integrally closed in $T / Z$, where $Z=\operatorname{Rad}(T)=\operatorname{Rad}(R) ; R / Z$ and $T / Z$ have the same total quotient ring; for each $p^{\prime} \in \operatorname{Spec}(T)$ it holds that $T_{p^{\prime}}=R_{p^{\prime} \cap R}$; if $\bar{p} \in \operatorname{Spec}(R / Z)$ is lost in $T / Z$, then $\bar{p}$ is a height one maximal ideal, $(R / Z)_{\bar{p}}$ is a discrete valuation ring, and $(T / Z)_{(R / Z)-\bar{p}}$ is its quotient field; and, for each $\bar{c} / \bar{b} \in(T / Z-R / Z$ ) (with $\bar{b}, \bar{c} \in R / Z$ and $\bar{b}$ regular in $R / Z$ ) the only prime ideals in $R / Z$ that contain $\bar{b}(R / Z)$ are height one maximal ideals, and if $\bar{c}$ is regular in $R / Z$, then $\bar{b} / \bar{c} \in R / Z$, so $(R / Z)[\bar{c} / \bar{b}]$ is a localization of $R / Z$.

PROOF. The proof of (2.1) (together with the result in [8] (concerning $A: B$ and mentioned preceding this remark) shows that if (a) does not hold, then for each $t \in T$ it holds that $R / Z$ and $(R / Z)[t]$ have the same total quotient ring, where $Z=\operatorname{Rad}(R)=\operatorname{Rad}(R[t])$, that $R$ is integrally closed in $R[t]$, and that $R / Z$ is integrally closed in $(R / Z)[\bar{t}]$, so it follows that $Z=\operatorname{Rad}(T)$, that $R / Z$ and $T / Z$ have the same total quotient ring, that $R$ is integrally closed in $T$, and that $R / Z$ is integrally closed in $T / Z$. Also, if $p^{\prime}<P^{\prime}$ are prime ideals in $T$ such that $p^{\prime} \cap R=P^{\prime} \cap R$, then $p^{\prime} \cap R[t]<P^{\prime} \cap R[t]$, where $t \in\left(P^{\prime}-p^{\prime}\right)$, so the fifth paragraph of the proof of (2.1) shows that (a) holds. Therefore if (a) does not hold, then each $p^{\prime} \in \operatorname{Spec}(T)$ is isolated over $p \cap R$, so $T_{p^{\prime}}=R_{p^{\prime} \cap R}$, since it follows from [2] that for each finite subset $U$ of $T$ it holds that $R_{p^{\prime} \cap R}=R[U]_{p^{\prime} \cap R[U]}$. Therefore, if $p \in \operatorname{Spec}(R)$ is lost in $T$, then each prime ideal $P$ in $R$ containing $p$ is also lost in $T$, since otherwise $R_{P}=T_{R-P}$, so $p^{\prime}=p R_{P} \cap T$ lies over $p$. Thus if $\bar{p}=p / Z$ is not a height one maximal ideal, then by the eighth paragraph of the proof of 
(2.1) it follows that (a) holds. If $\bar{p}$ is a height one maximal ideal in $R / Z$ which is lost in $T / Z$, then the eighth paragraph of the proof of (2.1) also shows that $(R / Z)_{\bar{p}}<(T / Z)_{(R / Z)-\bar{p}}$ and $(R / Z)_{\bar{p}}$ is integrally closed in $(T / Z)_{(R / Z)-\bar{p}}$. Thus, since altitude $\left((R / Z)_{\bar{p}}\right)=1$ and $R / Z$ is Noetherian, it follows that $(R / Z)_{\bar{p}}$ is a discrete valuation ring and $(T / Z)_{(R / Z)-\bar{p}}$ is its quotient field. Finally, since (a) does not hold, it follows as in the eighth paragraph of the proof of (2.1) that the last two statements in (b) concerning $\bar{b} / \bar{c}$ hold. Q.E.D.

(2.3) COROLlaRY. If $R<T$ are Noetherian rings and $P \in \operatorname{Spec}(R)$ is such that $R_{P}<T_{R-P}$, then there exists a ring $B$ finitely generated over $R$ and contained in $T$ with a prime ideal $P^{\prime}$ maximal (in $B$ ) with respect to lying over $P$ such that $\operatorname{grade}\left(P^{\prime} B_{P^{\prime}}\right) \leq 1$.

Proof. Since $R_{P}<T_{R-P}$, by $(2.1)$ there exists a ring $C$ that is finitely generated over $R_{P}$, that is contained in $T_{R-P}$, and that has a maximal ideal $Q$ such that grade $\left(Q C_{Q}\right) \leq 1$ and $Q \cap R_{P}=P R_{P}$. Therefore there exists a ring $B$ that is finitely generated over $R$ and that is contained in $T$ such that $C=B_{R-P}$. The conclusion readily follows from this. Q.E.D.

(2.4) REMARK. It follows immediately from (2.3) that:

(2.4.1) If $T$ is integral over $R$, then there exists a finite $R$-algebra $B$ contained in $T$ with a prime ideal $P^{\prime}$ lying over $P$ such that grade $\left(P^{\prime}\right) \leq 1$.

(2.4.2) If $T$ is an integral domain and $P \neq(0)$, then $\operatorname{grade}\left(P^{\prime}\right)=1$.

This section will be closed with one application of (2.1). Namely, it follows immediately from (2.1) that the answer to the question in [13, (6.7.1)] (problem (a) in the introduction) is no. The following specific example is included since it seems interesting to me. (The example resulted from some joint work with Steve McAdam; I am indebted to him for several helpful conversations concerning the contents of this paper and, in particular, for permission to use this example here.)

(2.5) EXAMPLE. There exists a Cohen-Macaulay local domain $(R, M)$ of altitude two such that: (a) $R^{\prime}$, the integral closure of $R$, is local and CohenMacaulay; (b) $\operatorname{Spec}(R) \cong \operatorname{Spec}\left(R^{\prime}\right)$; (c) there exists an infinite chain of local domains $B_{1}<C_{1}<B_{2}<C_{2}<\cdots$ between $R$ and $R^{\prime}$ such that each $C_{i}$ is Cohen-Macaulay; (d) there exists $X \in R$ such that $P^{\prime}=X R^{\prime}$ is a principal prime ideal and $X C_{i}=P^{\prime} \cap C_{i}$ for $i=0,1, \ldots$, where $C_{0}=R$; and, (e) $M^{\prime} \cap B_{i}$ is an imbedded prime divisor of $X B_{i}$ for $i=1,2, \ldots$, where $M^{\prime}$ is the maximal ideal in $R^{\prime}$.

ProOF. Let $(L, N)$ be an altitude one local domain such that $L^{\prime}$ is local with maximal ideal $N^{\prime}$ and $L^{\prime}$ is not a finite $L$-module, let $X$ be an indeterminate, and let $R_{0}=L[X]_{(N, X)}$. Then it is clear that $R_{0}$ is a Cohen-Macaulay local domain of altitude two, and so $R_{0}^{\prime}=L^{\prime}[X]_{\left(N^{\prime}, X\right)}$ is also, by [10, (25.13)]. Also, it is shown in $[4,(23.2 .5)]$ that there exists a finite integral extension domain $D$ of $R_{0}$ such that $D \subseteq R_{0}^{\prime}$ and such that $\operatorname{Spec}(D) \cong \operatorname{Spec}\left(R^{\prime}\right)$.

It is clear that $R_{0}$ is unmixed, so $D$ is also, by [9, Corollary, p. 61] (since $D$ is finitely generated over $R$ ) and so $D^{(1)}=\bigcap\left\{D_{p} ; p\right.$ is a height one prime ideal in $\left.D\right\}$ is a finite $D$-module, by [11, Lemma 5.11(1)]. Also, $D^{(1)} \subseteq R_{0}^{\prime}$, so $D^{(1)}$ is local, and $\left\{P \in \operatorname{Spec}\left(D^{(1)}\right) ; \operatorname{grade}(P)=1\right\}$ is the set of height one prime ideals in $D^{(1)}$, by $[11$, Lemma $5.6(2)]$. Therefore $\operatorname{grade}\left(M^{\prime} \cap D^{(1)}\right)>1$, so since altitude $\left(D^{(1)}\right)=$ 2 it follows that $D^{(1)}$ is Cohen-Macaulay. Let $R=D^{(1)}$. Let $P^{\prime}=X R^{\prime}$ and 
$M^{\prime}=\left(N^{\prime}, X\right) R^{\prime}$, so $M^{\prime}$ is the maximal ideal in $R^{\prime}$ and $P^{\prime} \cap R=X R$ (since $P^{\prime} \cap R$ is the radical of $X R$ (hence $X R$ is $P^{\prime} \cap R$-primary) and since $\left(R_{0}\right)_{X R_{0}}=R_{P^{\prime} \cap R}=$ $\left.R_{P^{\prime}}^{\prime}\right)$.

Let $R=C_{0}$ and assume that for some $i \geq 0$ the local rings $C_{0}<B_{1}<C_{1}<$ $\cdots<B_{i}<C_{i}$ have been constructed as in (c) (e). Then by (2.4.1) and (2.4.2) applied to $R=C_{i}, T=R^{\prime}$, and $P=M_{i}$, where $M_{i}$ is the maximal ideal in $C_{i}$, there exists a finite $C_{i}$-module $B_{i+1}$ contained in $R^{\prime}$ with a grade one maximal ideal. Then $B_{i+1}$ is local, so $\operatorname{grade}\left(M^{\prime} \cap B_{i+1}\right)=1$, so $M^{\prime} \cap B_{i+1}$ is an imbedded prime divisor of $X B_{i+1}$. Let $C_{i+1}=\left(B_{i+1}\right)^{(1)}$, so it follows as in the preceding paragraph for $D^{(1)}$ that $C_{i+1}$ is a finite $B_{i+1}$-module, a Cohen-Macaulay local domain contained in $R^{\prime}$, and $X C_{i+1}=P^{\prime} \cap C_{i+1}$. The conclusions readily follow from this. Q.E.D.

3. Persistent imbedded prime divisors. In [5, Appendix] a prime ideal $P$ such that $P \in \operatorname{Ass}\left(R / I^{k}\right)$ for all large $k$ was called a persistent prime divisor of $I$. With this terminology, in this section question (b) mentioned in the introduction is briefly considered: if $P_{1}, \ldots, P_{g}$ are regular prime ideals in a Noetherian ring $R$, then does there exist an ideal $I$ such that the persistent prime divisors of $I$ are exactly the ideals $P_{1}, \ldots, P_{g}$ ? By letting $I=P_{1} \cdots P_{g}$ and noting that $I^{n} R_{P_{i}}: P_{i} R_{P_{i}}>I^{n} R_{P_{i}}$ for $i=1, \ldots, g$ and for all $n \geq 1$ it follows that the $P_{i}$ are among the persistent prime divisors of $P_{1} \cdots P_{g}$. However, there may be other persistent prime divisors, as is shown in the following example.

(3.1) EXAMPLE. Let $(R, M)$ be a local domain of altitude $n \geq 2$ whose completion $R^{*}$ has a depth one prime divisor $z$ of zero and let $p$ be a height one prime ideal in $R$. (For example, $R$ could be the ring in [10, Example 2, pp. 203-205] in the case $m=0$.) Then there does not exist an ideal $I$ in $R$ whose only persistent prime divisor is $p$.

PROOF. Since $\operatorname{depth}(z)=1$ it follows that $I R^{*}+z$ is $M R^{*}$-primary for all nonzero ideals $I$ in $R$. Therefore it follows from [12, (2.9.1)] that $M \in \operatorname{Ass}\left(R / I^{k}\right)$ for all large $k$. Q.E.D.

Since it is easy to find an ideal with the $P_{i}$ among its persistent prime divisors, in the remainder of this section the following more difficult problem is considered: If $I$ is a regular ideal contained in $\bigcap\left\{P_{i} ; i=1, \cdots, g\right\}$, then does there exist an ideal $J$ integrally dependent on $I$ such that the $P_{i}$ are among the persistent prime divisors of $J$. (This problem was suggested by the rather deep result in [7], where it is shown that often, but not always, there exist a positive integer $m$ and an ideal $K$ in $R$ such that $I^{m} \subseteq K \subseteq\left(I^{m}\right)_{a}$ such that $\operatorname{Ass}\left(R / K^{n}\right)=E(I) \cup\left\{P_{1}, \ldots, P_{g}\right\}$ for all $n \geq 1$, where $E(I)$ is the set of essential prime divisors of $I$; the proof of this result is long and quite technical, but the proof of the problem considered in this section is quite easy.) As phrased, the answer to this question is no; for example, if $(R, M)$ is an integrally closed local domain of altitude at least two and $I=b R$ is a nonzero principal ideal in $R$, then $I$ is the only ideal that is integrally dependent on $I$, so $M \notin \operatorname{Ass}(R / J)$ for all ideals $J$ integrally dependent on $I$. However, it is shown in (3.2) that there always exists a free quadratic integral extension ring $B$ of $R$ such that $\operatorname{Spec}(B) \cong \operatorname{Spec}(R)$ and for which the answer is yes. To prove this result, two definitions and three facts concerning them are needed.

If $I$ is a regular ideal in a Noetherian $\operatorname{ring} R$, then $I_{a}$ denotes the integral closure in $R$ of $I$, and $I^{*}=\bigcup\left\{I^{n+1}: I^{n} ; n \geq 1\right\}$. Then: (a) it is shown in [6, Lemma 
8.2(vi)] that $I^{*}$ is an ideal such that $I \subseteq I^{*} \subseteq I_{a}$; (b) it is readily seen that if $A$ is a flat $R$-algebra, then $I^{*} A=(I A)^{*}$; and, (c) it is shown in [6, Lemma 8.3] that if $R$ is local with maximal ideal $M$ and if $J$ is an ideal in $R$ such that $I \subseteq J \nsubseteq I^{*}$, then $M \in \operatorname{Ass}\left(R /(I+M J)^{k}\right)$ for all $k \geq 1$. (If $I \nsubseteq J$, then $I \subseteq I+J$ and $I+M J=I+M(I+J)$, so the assumption that $I \subseteq J$ is not essential.)

(3.2) PROPOSITION. If $R$ is a Noetherian ring, then there exists a free quadratic integral extension ring $B$ of $R$ such that $\operatorname{Spec}(B) \cong \operatorname{Spec}(R)$ and such that the following hold:

(3.2.1) If $P_{1}, \ldots, P_{g}$ are prime ideals in $R$ and if $I$ is any regular ideal in $R$ contained in $\bigcap\left\{P_{i} ; i=1, \ldots, g\right\}$, then there exists an ideal $J$ in $B$ integrally dependent on $I B$ such that the $g$ prime ideals in $B$ that correspond to $P_{1}, \ldots, P_{g}$ are in $\operatorname{Ass}\left(B / J^{k}\right)$ for all $k \geq 1$.

(3.2.2) If $\mathbf{P}^{\prime}=\left\{Q_{1}, \ldots, Q_{g}\right\}$ is a finite set of prime ideals in $B$ and if $H$ is any regular ideal in $B$ contained in $\bigcap\left\{Q_{i} ; Q_{i} \in \mathbf{P}^{\prime}\right\}$, then there exists an ideal $J$ in $B$ such that $J_{a}=H_{a}$ and $\mathbf{P}^{\prime} \subseteq \operatorname{Ass}\left(B / J^{k}\right)$ for all $k \geq 1$.

ProOF. Let $B=R[X] / X^{2} R[X]$, so $B$ is a free quadratic integral extension ring of $R$. Let $x=X+X^{2} R[X]$, let $Q$ be a prime ideal in $B$, and let $P=Q \cap R$. Then it is readily seen that $Q=(P, x) B$, so it follows that $\operatorname{Spec}(B) \cong \operatorname{Spec}(R)$.

To prove (3.2.1) let $Q_{i}=\left(P_{i}, x\right) B$ for $i=1, \ldots, g$, so $Q_{i}$ is the prime ideal in $B$ that corresponds to $P_{i}$. Then it is clear that if $Q_{i}$ is a minimal prime divisor of $I B$, then $Q_{i}$ is a minimal prime divisor of all ideals which are integrally dependent on $I B$, so it may be assumed that no $Q_{i}$ is a minimal prime divisor of $I B$. Let $J=I B+$ $Q_{1} \cdots Q_{g}(I B)_{a}$, so $J$ is integrally dependent on $I B$. Fix $i=1, \ldots, g$, let $Q=Q_{i}$, and let $K$ be the product of the $Q_{j}(j=1, \ldots, g)$ which are properly contained in $Q\left(K=B\right.$, if there are no such $\left.Q_{j}\right)$. Assume it is known that $K_{Q}\left(I B_{Q}\right)_{a} \nsubseteq$ $\left(I B_{Q}\right)^{*}$. Then since $J B_{Q}=I B_{Q}+Q_{Q}\left(K_{Q}\left(I B_{Q}\right)_{a}\right)$, it follows from (c) that $Q B_{Q} \in$ $\operatorname{Ass}\left(B_{Q} /\left(J B_{Q}\right)^{k}\right)$ for all $k \geq 1$, so $Q \in \operatorname{Ass}\left(B / J^{k}\right)$ for all $k \geq 1$ and for all $Q \in$ $\left\{Q_{1}, \ldots, Q_{g}\right\}$, as desired. Therefore it remains to show that $K_{Q}\left(I B_{Q}\right)_{a} \nsubseteq\left(I B_{Q}\right)^{*}$.

For this, suppose, on the contrary, that $K_{Q}\left(I B_{Q}\right)_{a} \subseteq\left(I B_{Q}\right)^{*}$, let $q$ be a minimal prime divisor of $I B$ which is (properly) contained in $Q$, and let $p=q \cap R$. Then it follows that $\left(I B_{q}\right)_{a}=\left(K_{Q}\left(I B_{Q}\right)_{a}\right) B_{q} \subseteq\left(I B_{Q}\right)^{*} B_{q}$. However, it is clear that $B_{q}=R_{p}[x]$ is a free quadratic integral extension ring of $R_{p}$, so it follows from (b) that $\left(I B_{Q}\right)^{*} B_{q}=\left(I R_{p}\right)^{*} B_{q}$, and so $x \notin\left(I B_{Q}\right)^{*} B_{q}$. But it is clear that $x \in\left(I B_{q}\right)_{a}$, so $x \in\left(\left(I B_{q}\right)_{a}-\left(I B_{Q}\right)^{*} B_{q}\right)$, and this contradicts the just noted containment. Therefore $K_{Q}\left(I B_{Q}\right)_{a} \nsubseteq\left(I B_{Q}\right)^{*}$.

For (3.2.2) let $b=r+s x \in H$, where $r, s \in R$. Now $x \in H_{a}$, so $r \in H_{a} \cap R=$ (say) I. Therefore $H \subseteq(I, x) B \subseteq H_{a}$, and $I B \subseteq(I, x) B \subseteq(I B)_{a}$, so $(I B)_{a}=H_{a}$. The conclusion follows from this and (3.2.1), since $I$ is regular, $I \subseteq \bigcap\left\{P_{i} ; i=1, \ldots, g\right\}$, where $P_{i}=Q_{i} \cap R$, and $Q_{i}=\left(P_{i}, x\right) B$. Q.E.D.

\section{REFERENCES}

1. L. Dechene, Adjacent extensions of rings, Ph. D. Dissertation, Univ. of California, Riverside, 1978.

2. E. G. Evans, Jr., A generalization of Zariski's Main Theorem, Proc. Amer. Math. Soc. 26 (1970), 45-48.

3. D. Ferrand and J.-P. Oliver, Homomorphismes minimaux d'anneaux, J. Algebra 16 (1970), 461-471. 
4. A. Grothendieck, Elements de geometrie algebrique. IV (Premiere Partie), Inst. Hautes Études Sci., Paris, 1964.

5. D. Katz, S. McAdam, J. Okon, and L. J. Ratliff, Jr., Essential prime divisors and projectively equivalent ideals, J. Algebra (to appear).

6. S. McAdam, Asymptotic prime divisors, Lecture Notes in Math., vol. 1023, Springer-Verlag, Berlin and New York, 1983.

7. S. McAdam and L. J. Ratliff, Jr., Persistent primes and projective extensions of ideals, Rocky Mountain J. Math (to appear).

8. M. L. Modica, Maximal subrings, Ph. D. Dissertation, Univ. of Chicago, 1975.

9. M. Nagata, On the chain problem of prime ideals, Nagoya Math. J. 10 (1956), 51-64.

10. __ Local rings, Interscience Tracts 13, Interscience, New York, 1962.

11. L. J. Ratliff, Jr., On quasi-unmixed local domains, the altitude formula, and the chain condition for prime ideals (II), Amer. J. Math. 92 (1970), 99-144.

12. _ Independent elements, integrally closed ideals, and quasi-unmixedness, J. Algebra 73 (1981), 327-343.

13. , Five notes on asymptotic prime divisors, Math. Z. 190 (1985), 567-581.

14. O. Zariski and P. Samuel, Commutative algebra, Vol. I, Van Nostrand, New York, 1958.

15. __ Commutative algebra, Vol. II, Van Nostrand, New York, 1960.

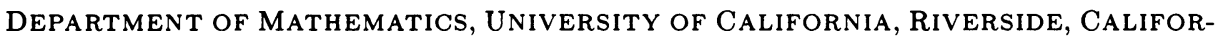
NIA 92521 